\title{
Síntese, caracterização e atividade antitumoral in vitro de uma série de 1,2,4-oxadiazois 3,5-substituídos
}

Synthesis, characterization and in vitro antitumor activity of a series of 3,5-substituted 1,2,4oxadiazoles

\author{
E. E. da Silva ${ }^{1}$; B. M. M. G. Aciolii ${ }^{1}$; M. M. S. Mata ${ }^{1}$; G. C. G. Militão ${ }^{4}$; T. G. da \\ Silva $^{4}$; J. J. R. de Freitas ${ }^{2}$; J. C. R. Freitas ${ }^{3}$; J. R. de Freitas Filho ${ }^{1 *}$ \\ ${ }^{1}$ Departamento de Química, Universidade Federal Rural de Pernambuco, 52171-900, Recife-PE, Brasil \\ ${ }^{2}$ Unidade Acadêmica do cabo de Santo Agostinho, Universidade Federal Rural de Pernambuco, 54518-430, Cabo-PE, \\ Brasil \\ ${ }^{3}$ Centro de Educação e Saúde, Universidade Federal de Campina Grande, 58175-000, Cuité-PB, Brasil \\ ${ }^{4}$ Departamento de Fisiologia e Farmacologia, Universidade Federal de Pernambuco, 506670-901, Recife-PE, Brasil
}

*joaoveronice@yahoo.com.br

(Recebido em 05 de outubro de 2020; aceito em 23 de novembro de 2020)

\begin{abstract}
Neste trabalho, é descrito a síntese, caracterização estrutural e atividade antitumoral in vitro de 1,2,4oxadiazois. As arilamidoximas, produtos de partida de interesse, foram obtidos em bons rendimentos (7092\%). Por outro lado, os 3-aril-(1,2,4-oxadiazol-5-il)-propan-2-ona (5a-c) foram obtidos a partir do acetoacetato de etila com arilamidoxima através do aquecimento durante 4 horas em rendimento de 74 $88 \%$. Em seguida os compostos 3a-c foram submetidos a reação de redução com borohidreto de sódio para fornecer os 3-aril-(1,2,4-oxadiazol-5-il)-propan-2-ol (6a-c) em rendimento variando de 75-88\%. Compostos contendo o núcleo oxadiazolico da série (5a-c) e (6a-c) foram submetidos a teste biológico para verificação da citoxicidade in vitro dos mesmos frente a células tumorais - HT29 (carcinoma de cólon - humano), HL60 (leucemia promielocítica) e NCI H-292 (câncer de pulmão - humano). Os compostos (5a-c) e (6a-c) tiveram suas atividades antitumoral in vitro testada e $\mathbf{5 a} / \mathbf{b}$ apresentaram atividade moderada e $\mathbf{5} \mathbf{c}$ boa atividade frente as células HL60. Por outro lado, os compostos 6a-c não apresentaram atividades.
\end{abstract}

Palavras-chave: oxadiazol, citotoxidade, antitumoral

In this work, the synthesis, structural characterization and in vitro antitumor activity of 1,2,4-oxadiazoles is described. Arylamidoximes, starting products of interest, were obtained in good yields (70-92\%). On the other hand, 3-aryl-(1,2,4-oxadiazol-5-yl)-propan-2-one (5a-c) were obtained from ethyl acetoacetate with arylamidoxime by heating for 4 hours in yield of $74-88 \%$. Then compounds 3a-c were subjected to a reduction reaction with sodium borohydride to provide 3-aryl-(1,2,4 -oxadiazol-5-yl)-propan-2-ol (6a-c) in yield ranging from 75 to $88 \%$. Compounds containing the oxadiazole nucleus of the series (5a-c) and (6a-c) were subjected to biological testing to verify their in vitro cytoxicity against tumor cells - HT29 (colon carcinoma - human), HL60 (promyelocytic leukemia) and NCI H-292 (lung cancer - human). The compounds (5a-c) and (6a-c) had their antitumor activities tested in vitro and $\mathbf{5 a} / \mathbf{b}$ showed moderate activity and $\mathbf{5 c}$ good activity against HL60 cells. On the other hand, compounds $\mathbf{6 a - c}$ showed no activity.

Keywords: oxadiazole, cytotoxicity, antitumor

\section{INTRODUÇÃO}

Compostos heterocíclicos têm aplicações em diversos campos, tais como na indústria farmacêutica, na agricultura e na química de materiais. Heterocíclicos de estrutura rígida com cinco membros constituídos por dois átomos de carbono, dois átomos de nitrogênio e um átomo de oxigênio, são chamados de oxadiazóis. Dentre as classes de compostos heterocíclicos contendo anéis de cinco membros, o 1,2,4-oxadiazol é considerado um dos menos aromáticos com índice de aromaticidade de $I_{5}=39$ ou $I_{A}=48$ [1].

A reatividade deste núcleo é uma consequência da ligação $\mathrm{O}-\mathrm{N}$ ser fraca, dos carbonos $\mathrm{C}(3)$ e $\mathrm{C}(5)$ ter caráter eletrofílico, podendo, a reatividade deste último, ser aumentada pela presença de substituintes retiradores de elétrons. Outras explicações são o caráter nucleofílico do N(4), o 
caráter ambifílico de $\mathrm{N}(2)$, a capacidade do oxigênio de atuar como um bom grupo de saída interno, e a presença de uma cadeia lateral que possa estar envolvida nos rearranjos intramoleculares. Estas características tornam este heterociclo multifuncional (Figura 1), cuja reatividade depende fortemente do substituinte, dos reagentes e condições reacionais utilizados [2]. Por outro lado, observa-se que o nitrogênio $\mathrm{N}(2)$ tem um caráter ambifílico, podendo ser alvo de vários rearranjos intramoleculares, dependendo da cadeia lateral ligada ao carbono $\mathrm{C}(3)$.

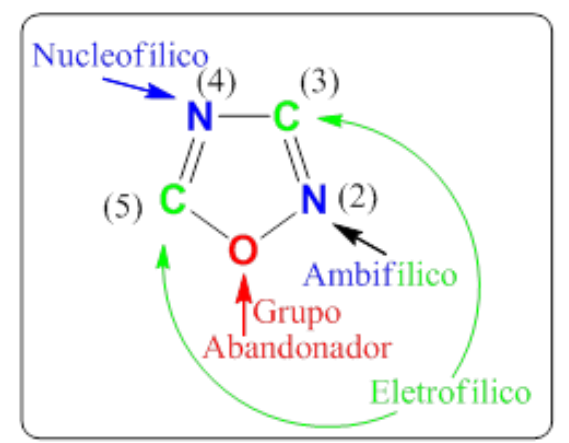

Figura 1: Reatividade do 1,2,4-oxadiazol [2].

A descoberta de 1,2,4-oxadiazóis de origem natural abriu novo caminho para síntese total e modificação estrutural destas fidianidinas [3]. Este fato levou a necessidade do desenvolvimento de novas metodologias sintéticas para obtenção destes heterociclos em altos rendimentos e em menor tempo de reação [4]. Devido à grande importância dos 1,2,4-oxadiazóis, neste trabalho, apresentamos alguns tópicos recentes sobre suas metodologias de síntese e aplicações tanto na área de materiais como em química medicinal.

Nos últimos 40 anos, o 1,2,4-oxadiazol heterociclo tem sido amplamente explorado trazendo um grande número de compostos exibindo diversas atividades biológicas. A literatura relata um conjunto diversificado de aplicações (Figura 2) para o núcleo de 1,2,4-oxadiazol na química medicinal [5, 6], como antiasmático (1) [7], antidiabético (2) [8], antitumoral $(\mathbf{3}, 4$ e 5) [9, 10, 11], anti-inflamatório (6) [12], antioxidante (7) [13] antimicrobiano (8 e 9) [14], anti-Alzheimer (10) $[15,16]$. Além disso, derivados de 1,2,4-oxadiazol também encontraram aplicação como cristais líquidos (11) supramoleculares e HEDMs [17, 18, 19].

Por outro lado, os 1,2,4-oxadiazóis também podem ser utilizados para fins agrícolas. Alguns derivados oxadiazólicos apresentaram boa atividade acaricida e inseticida (13) e fungicida [20]. Em 2009, Neves Filho et al. (2009) [21] relataram a atividade larvicida do composto (12) contra larvas L4 de Aedes aegypti (Figura 2). Recentemente, em um bioensaio preliminar, Yang et al. (2020) [22] demonstrou que o composto (14) apresentou boas atividades larvicidas contra larvas de mosquito a $10 \mathrm{mg} / \mathrm{L}$ e também boas atividades fungicidas. 


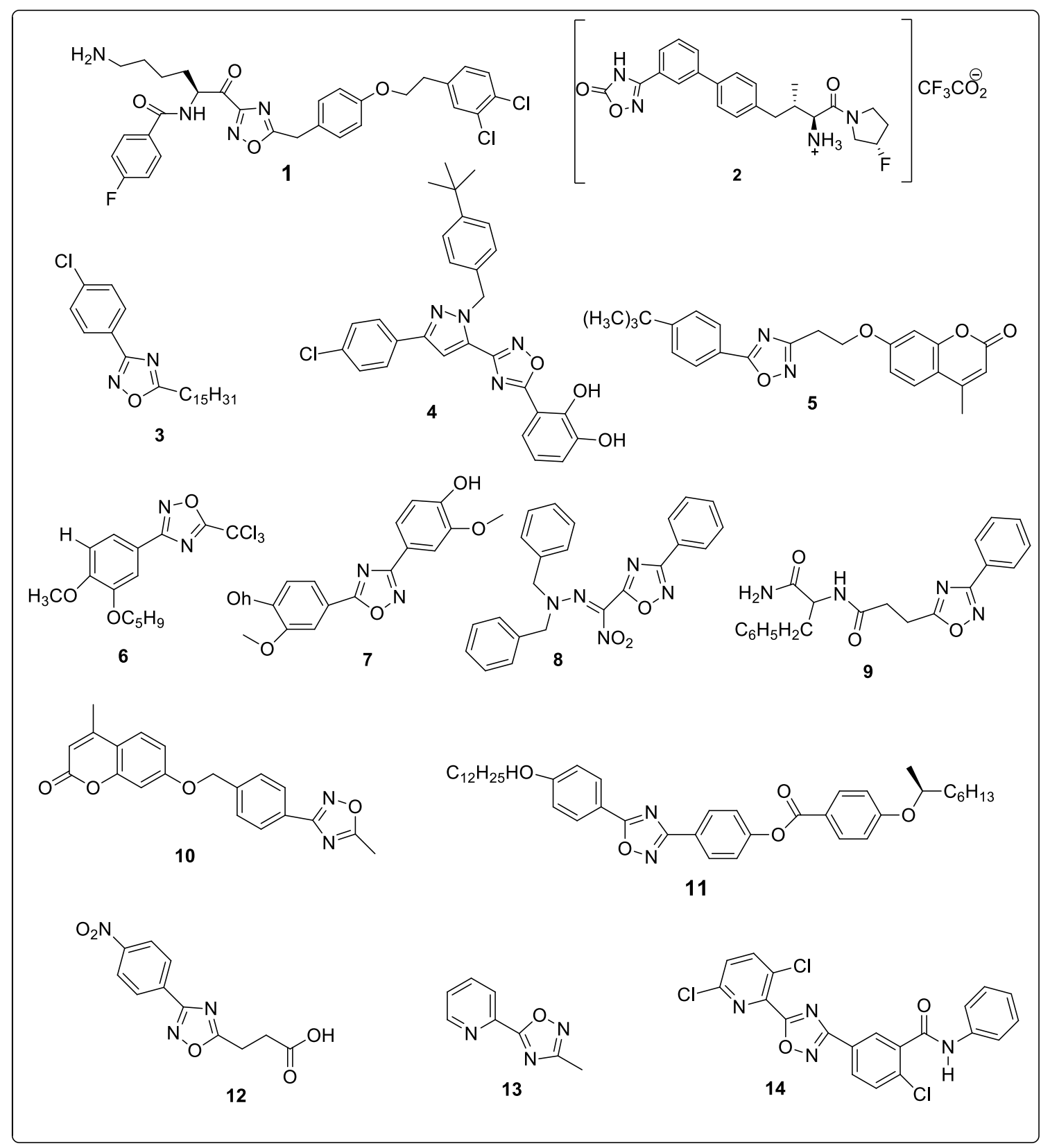

Figura 2: Exemplos de 1,2,4-Oxadiazóis com aplicações na química medicinal, química de materiais e na química agrícola [7-13, 20-22].

Em geral, a síntese de 1,2,4-oxadiazol envolve a reação de ciclocondensação entre amidoxima e ácidos carboxílicos, ou anidridos de ácido, ou halogenetos de ácido, ou ésteres de ácido carboxílico, ou cicloadição 1,3-dipolar de nitrilas com $N$-óxidos de nitrila [2]. No nosso trabalho optamos pela rota mais comum que consiste no acoplamento das amidoximas com derivados de ácido carboxílico ativados, no caso os ésteres. Geralmente o método descrito na literatura utiliza ácidos carboxílicos e/ou halogenetos de ácido, solvente e base, e o produto final é obtido com elevado tempo de duração. Na nossa proposta utilizamos uma síntese "one-pot" e essa tem demonstrado ser uma metodologia rápida e eficiente na síntese de 1,2,4-oxadiazóis 3,5dissusbstitídos. Outro ponto de destaque na nova metodologia e segue um dos princípios da química verde, ou seja, a economia de átomos e geração de pouco resíduos.

Mediante ao exposto, e consciente do papel dos 1,2,4-oxadiazol quer seja na química medicinal ou química dos materiais, este trabalho tem como objetivo descrever a síntese, a caracterização estrutural e a atividade antitumoral in vitro de 1,2,4-oxadiazois 3,5-dissubstitídos. 


\section{MATERIAL E MÉTODOS}

\subsection{Materiais}

Na preparação dos compostos foi utilizado reagente e solvente na sua forma comercial, p.a., dos fornecedores Merck, Aldrich, Vetec e Cinética, sem nenhuma purificação adicional (só quando for mencionado), como foi o caso dos solventes hexano e acetato de etila que indicam presença de agentes contanimantes. O acompanhamento das reações foi realizado através de cromatografia em camada delgada (CCD), onde foi empregada placas prontas de sílica-gel contendo indicador fluorescente $\mathrm{F}_{254}$. Os solventes usados como fase móvel foi o sistema de $\mathrm{CH}_{2} \mathrm{Cl}_{2}$ /AcOEt (9:1). Para visualização dos compostos foi usada lâmpada de ultravioleta e/ou cuba contendo sistema de $\mathrm{H}_{2} \mathrm{SO}_{4} / \mathrm{EtOH}(9,5: 0,5)$. Para cromatografia em coluna foi utilizada sílica-gel 60 (Merck, 70 - 230 mesh). Os espectros de infravermelho com transformada de Fourier foram obtidos com o equipamento Varian modelo 640 FTIR. Os espectros de RMN ${ }^{1} \mathrm{H}$ e ${ }^{13} \mathrm{C}$ foram obtidos nos equipamentos da Varian modelos Unitty Plus (300 MHz para hidrogênio e $75 \mathrm{MHz}$ para carbono) e UNMRS (400 MHz para hidrogênio e $100 \mathrm{MHz}$ para carbono), usando clorofórmio deuterado e tetrametilsilano (TMS) como padrão interno. Os valores do deslocamento químico $(\delta)$ estão expressos em partes por milhão $(\mathrm{ppm})$ e as constantes de acoplamento $(J)$ em Hertz $(\mathrm{Hz})$. Os pontos de fusão serão realizados no Eletro-thermal série IA 9100 Digital Metlting Point.

\subsection{Métodos}

\section{a) Sintese das arilamidoximas}

Em um pote de penicilina, a nitrila $(1 \mathrm{mmol})$ foi dissolvida em $5 \mathrm{~mL}$ de álcool etílico. Separadamente, em outro recipiente de penicilina, foram dissolvidos $3 \mathrm{mmol}$ de cloridrato de hidroxalamina e $1,5 \mathrm{mmol}$ de carbonato de sódio em $5 \mathrm{~mL}$ de água. Esta solução aquosa foi adicionada a solução alcoólica contendo a nitrila e o meio reacional foi levado a agitação por aproximadamente 24 horas, até o consumo total da nitrila, acompanhado através de cromatografia em camada delgada (CCD), em sistema $\mathrm{CH}_{2} \mathrm{Cl}_{2}$ : AcOEt (9:1) Após término da reação, o etanol foi evaporado e duas fases foram formadas, a fase aquosa e o produto insolúvel em água, onde foi realizada uma extração com acetato de etila $(2 \times 10 \mathrm{~mL})$, em seguida a fase orgânica foi secada com $\mathrm{Na}_{2} \mathrm{SO}_{4}$. Após uma filtração, o solvente foi removido sob pressão reduzida e o produto purificado por cristalização em clorofórmio. Os produtos 3a-c foram obtidos com rendimentos de $70-92 \%$.

\section{b) Síntese dos 1,2,4-oxadiazois}

Em um tubo de penicilina adicionou-se $0,4 \mathrm{~g}(2,5 \mathrm{mmol})$ da arilamidoxima e 0,644 $\mathrm{g}(3,9$ mmol) do acetoacetato de etila. Em seguida mistura-se os reagentes com o auxílio de uma espátula e leva-se a um banho de óleo que deve estar inicialmente pré-aquecido a $120^{\circ} \mathrm{C}$, durante 4 horas, nesse intervalo de tempo a temperatura pode variar de $120-140{ }^{\circ} \mathrm{C}$. A reação foi acompanhada através de cromatografia em camada delgada (CCD) em sistema de eluição 9:1 (diclorometano/acetato de etila). Após o término da reação comprovado por CCD e alcance da temperatura ambiente pelo produto realizou-se uma cromatografia em coluna em sistema de eluição hexano/acetato de etila, sendo o produto 5a-c obtido em rendimentos de 74-88\%. 
Caracterização dos compostos 5a-c

Composto 5a: 3-fenil-[1,2,4 -oxadiazol-5-il]-propan-2-ona

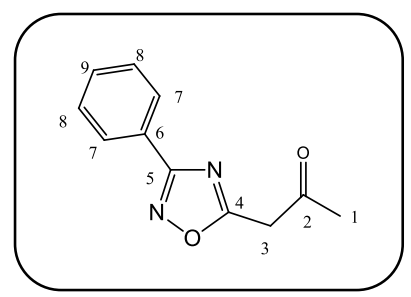

Semi-sólido amarelado, rendimento $88 \% ; \quad R_{\mathrm{f}}=0,80$ $\left(\mathrm{CH}_{2} \mathrm{Cl}_{2}\right.$ :AcOEt, 9:1); IV $v_{\max }(\mathrm{KBr}): 2926,1714,1363,1164 \mathrm{~cm}^{-1}$; $\mathrm{RMN}^{1} \mathrm{H}\left(300 \mathrm{MHz}, \mathrm{CDCl}_{3}\right): \delta 8,10-8,04(\mathrm{~m}, 2 \mathrm{H}, \mathrm{H} 7$ e H-8, Ph-H), 7,53-7,47 (m, 3H, H-9, H-4 e H-5, Ph-H), 4,10 (s, 2H, CH 2 ), 2,33(s, $\left.3 \mathrm{H}, \mathrm{CH}_{3}\right) ; \mathrm{RMN}{ }^{13} \mathrm{C}\left(75 \mathrm{MHz}, \mathrm{CDCl}_{3}\right): \delta 198,93(\mathrm{C}-2) ; 172,97(\mathrm{C}-$ 5); 168,56(C-4); 131,27(C-9); 128,82(C-8); 127,36(C-7); 126,35(C6); 41,51(C-3); 29,84(C-1).

Composto 5b: 3-p-toluil-[1,2,4 -oxadiazol-5-il]-propan-2-ona

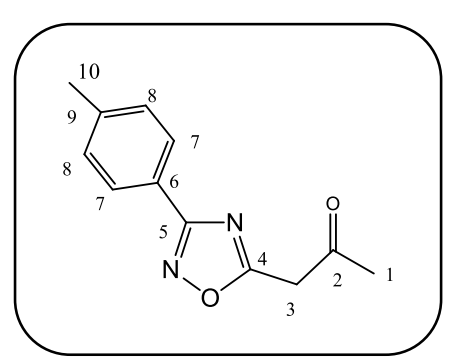

Semi-sólido amarelado, rendimento $74 \% ; \quad R_{\mathrm{f}}=0,72$ $\left(\mathrm{CH}_{2} \mathrm{Cl}_{2}\right.$ :AcOEt, 9:1); IV $v_{\max }(\mathrm{KBr}): 2924,1720,1592,1361$, $1169 \mathrm{~cm}^{-1}$; RMN ${ }^{1} \mathrm{H}\left(300 \mathrm{MHz}, \mathrm{CDCl}_{3}\right): \delta 8,00-7,78(\mathrm{~d}, 2 \mathrm{H}$, $J=8,1 \mathrm{~Hz} \mathrm{H} 7$ ); 7,21-7,18 (d, 2H, $J=8,1 \mathrm{~Hz} \mathrm{H8}$ );4,10 (s, 2H, $\left.\mathrm{CH}_{2}\right), 2,32\left(\mathrm{~s}, 3 \mathrm{H}, \mathrm{CH}_{3}\right) ; 2,25\left(\mathrm{~s} .3 \mathrm{H}, \mathrm{CH}_{3}\right), \mathrm{RMN}^{13} \mathrm{C}(75 \mathrm{MHz}$, $\left.\mathrm{CDCl}_{3}\right): \delta$ 199,01(C-2); 172,79(C-5); 168,54(C-4); 141,63(C-6); $129,48(\mathrm{C}-7) ; 127,27(\mathrm{C}-8) ; 123,50(\mathrm{C}-9) ; 41,50(\mathrm{C}-3) ; 29,82(\mathrm{C}-$ $10) ; 21,34(\mathrm{C}-1)$.

Composto 5c: 3-m-toluil-[1,2,4 -oxadiazol-5-il]-propan-2-ona

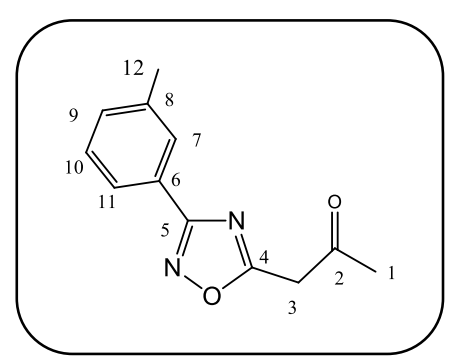

Semi-sólido amarelado, rendimento 83\%; $R_{\mathrm{f}}=0,85$ $\left(\mathrm{CH}_{2} \mathrm{Cl}_{2}\right.$ :AcOEt, 9:1); IV $v_{\max }(\mathrm{KBr}): 2965,1721,1732,1359$, $1166 \mathrm{~cm}^{-1} ; \mathrm{RMN}^{1} \mathrm{H}\left(300 \mathrm{MHz}, \mathrm{CDCl}_{3}\right): \delta$ 8,00-7,88 (d, $2 \mathrm{H}$, $J=8,7 \mathrm{~Hz} \mathrm{H7}$ ); 7,40-7,28 (m, 3H, $J=8,7 \mathrm{~Hz}) ; 4,10$ (s, 2H, $\mathrm{CH}_{2}$ ), $2,42\left(\mathrm{~s}, 3 \mathrm{H}, \mathrm{CH}_{3}\right) ; 2,35\left(\mathrm{~s} .3 \mathrm{H}, \mathrm{CH}_{3}\right) ; \mathrm{RMN}^{13} \mathrm{C}\left(75 \mathrm{MHz}, \mathrm{CDCl}_{3}\right)$ : $\delta \quad 198,91(\mathrm{C}-2) ; \quad 172,87(\mathrm{C}-5) ; \quad 168,63 \quad(\mathrm{C}-4) ; \quad 132,04(\mathrm{C}-9)$; 138,19(C-10), 128,46(C-6); 127,89 (C-8); 126,18(C-7); 41,50(C3); 29,81(C-12); 21,23(C-1).

\section{c) Reação de redução dos 1,2,4-oxadiazois}

Em um balão de fundo redondo de $100 \mathrm{~mL}$ foi dissolvido o composto $\mathbf{5 a - c}(1 \mathrm{mmol}) \mathrm{em}$ metanol $(15 \mathrm{~mL})$, em seguida foi adicionado o agente redutor boridreto de sódio à $0{ }^{\circ} \mathrm{C}$, deixando sob agitação por 30 minutos. Após o término da reação o solvente foi evaporado sob pressão reduzida. Em seguida a reação foi tratada com água $(40 \mathrm{~mL})$ e acetato de etila $(40 \mathrm{~mL})$. A fase orgânica foi secada com sulfato de sódio anidro. Em seguida foi feito uma filtração e o solvente foi removido sob pressão reduzida. Dando continuidade, o produto bruto foi submetido a uma cromatografia em coluna em sistema de eluição hexano/acetato de etila (7:3) fornecendo os produtos 6a-c em nos rendimentos de $75-88 \%$. 
Caracterização dos compostos 6a-c

Composto 6a: 3-fenil-[1,2,4 -oxadiazol-5-il]-propan-2-ol

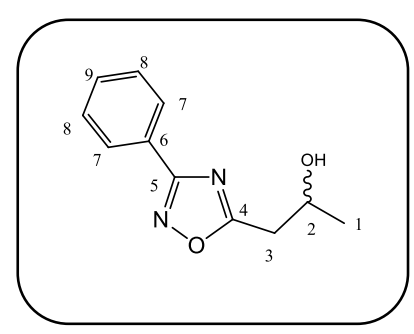

Óleo amarelado, rendimento $88 \% ; R_{\mathrm{f}}=0,55\left(\mathrm{CH}_{2} \mathrm{Cl}_{2}\right.$ :AcOEt, 9:1); IV $v_{\max }(\mathrm{KBr}): 3406,2973,1595,1367,1117 \mathrm{~cm}^{-1}$; RMN ${ }^{1} \mathrm{H}(300$ $\mathrm{MHz}, \mathrm{CDCl}_{3}$ ): $\delta$ 8,03-7,99 (m, 2H, H7 e H-8, Ph-H), 7,48-7,39 (m, $3 \mathrm{H}, \mathrm{H}-9, \mathrm{H}-4$ e H-5, Ph-H), 4,40 (m, 1H, CH), 3,41 (Banda larga referente ao $\mathrm{H}$ do $\mathrm{OH}) ; 3,08-2,95\left(\mathrm{~m}, 2 \mathrm{H}, \mathrm{CH}_{2}\right) ; 1,32\left(\mathrm{~S}, 3 \mathrm{H}, \mathrm{CH}_{3}\right)$; $1,30\left(\mathrm{~S}, 3 \mathrm{H}, \mathrm{CH}_{3}\right) ; \mathrm{RMN}^{13} \mathrm{C}\left(75 \mathrm{MHz}, \mathrm{CDCl}_{3}\right): \delta 178,12(\mathrm{C}-5)$; $168,24(\mathrm{C}-4) ; 131,56(\mathrm{C}-9)$ 129,13(C-8); 127,67(C-7); 126,65(C-6); 65,36(C-2);36,05(C-3) 23,07(C-1).

Composto 6b: 3-p-toluil-[1,2,4 -oxadiazol-5-il]-propan-2-ol

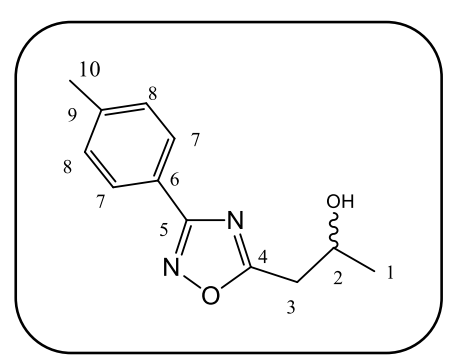

Óleo amarelado, rendimento 80\%; $R_{\mathrm{f}}=0,42\left(\mathrm{CH}_{2} \mathrm{Cl}_{2}: \mathrm{AcOEt}\right.$, 9:1); IV $v_{\max }(\mathrm{KBr}): 3406,2973,1580,1363,1114 \mathrm{~cm}^{-1}$; RMN ${ }^{1} \mathrm{H}\left(300 \mathrm{MHz}, \mathrm{CDCl}_{3}\right): \delta 7,84-7,82(\mathrm{~d}, 2 \mathrm{H}, J=8,1 \mathrm{~Hz}), 7,18-7,15$ (d, 2H,J=8,1); 4,40 (m, 1H, CH); 3,2(Banda larga referente ao $\mathrm{H}$ do $\mathrm{OH}), 3,08-2,95\left(\mathrm{~m}, 2 \mathrm{H}, \mathrm{CH}_{2}\right) ; 1,32\left(\mathrm{~S}, 3 \mathrm{H}, \mathrm{CH}_{3}\right) ; 1,30(\mathrm{~S}, 3 \mathrm{H}$, $\left.\mathrm{CH}_{3}\right)$; $\mathrm{RMN}{ }^{13} \mathrm{C}\left(75 \mathrm{MHz}, \mathrm{CDCl}_{3}\right): \delta 177,11(\mathrm{C}-5) ; 167,42(\mathrm{C}-4)$; 141,09(C-9); 129,13(C-8); 129,02(C-7); 126,76(C-6); 64,52(C2); 35,19(C-3) 22,79(C-10); 21,57(C-1).

Composto 6c: 3-m-toluil-[1,2,4 -oxadiazol-5-il]-propan-2-ol

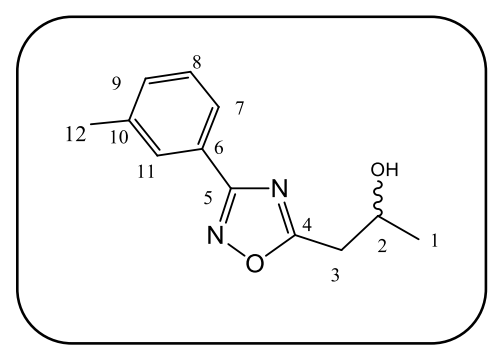

Óleo amarelado, rendimento $75 \% ; R_{\mathrm{f}}=0,60\left(\mathrm{CH}_{2} \mathrm{Cl}_{2}\right.$ : AcOEt, 9:1); IV $v_{\max }(\mathrm{KBr}): 3406,2973,1596,1367,1122 \mathrm{~cm}^{-1}$; RMN ${ }^{1} \mathrm{H}\left(300 \mathrm{MHz}, \mathrm{CDCl}_{3}\right): \delta$ 7,84- 7,82 (d, $\left.2 \mathrm{H}, J=8,1 \mathrm{~Hz}\right), 7,18-$ 7,15 (d, 2H, J=8,1), 4,40 (m, 1H, CH), 3,0 (Banda larga referente ao $\mathrm{H}$ do $\mathrm{OH}) ; 2,35\left(\mathrm{~s}, 3 \mathrm{H}, \mathrm{CH}_{3}\right) ; 1,26-1,24(\mathrm{~d}, 2 \mathrm{H}$, ${ }^{3} J=6,3 \mathrm{~Hz} \mathrm{CH}$ ); $\mathrm{RMN}^{13} \mathrm{C}\left(75 \mathrm{MHz}, \mathrm{CDCl}_{3}\right): \delta 177,68(\mathrm{C}-5)$; 168,00(C-4); 141,67(C-9) 129,59(C-8); 127,34(C-7); 123,58(C-6); 65,08(C-2); 35,75(C-3) 22,79(C-12); 21,57(C-1).

\section{d) Atividades antitumoral}

O potencial citotóxico dos 1,2,4-oxadiazóis foi realizado pelo método do MTT (Microcultura de Tetrazolium) utilizando 3 linhagens celulares (HL-60, HT 29 e NCL H-292). O ensaio consiste em uma análise colorimétrica baseada na redução do sal amarelo 3-(4,5-dimetil-2-tiazol) -2,5difenil-2-H-brometo de tetrazolium (MTT) em azul de formazan, descrito por Mosmann 1983 [23], pela atividade da enzima succinato desidrogenase presente na mitocôndria da célula viável, permitindo dessa maneira quantificar a porcentagem de células vivas. Esse método é utilizado no programa de screening do National Cancer Institute dos Estados Unidos (CI), que testa mais de 10.000 amostras a cada ano [24]. O estudo citotóxico pelo método do MTT permite definir facilmente a citotoxicidade, mas não o mecanismo de ação [25], sendo um método rápido, sensível e de baixo custo.

Para concentração única, uma escala de intensidade foi utilizada para avaliar o potencial citotóxico das amostras testadas. De acordo com o percentual de inibição de crescimento celular, as amostras podem ser classificadas em amostras sem atividade (1-20), com pouca atividade (2050), atividade moderada (50-70) e muita atividade numa escala de citotoxidade (70-100). Adicionalmente, os experimentos serão analisados segundo suas médias e respectivos desvios no 
programa Graphpad Prism, feitas também pelo laboratório de Departamento de Fisiologia e Farmacologia da UFPE. Cabe ressaltar que a avaliação de cada amostra foi testada em duplicata.

\section{RESULTADOS E DISCUSSÃO}

Inicialmente foram sintetizadas três arilamidoximas 3a-c. O método utilizado para obtenção das arilamidoximas, foi o clássico, ou seja, agitação em temperatura ambiente por 24 ou 48 horas. As diferentes arilamidoximas 3a-c foram sintetizadas a partir de arilnitrilas 1a-c com o cloridrato de hidroxilamina $\left(\mathrm{NH}_{2} \mathrm{OH}\right.$. $\left.\mathrm{HCl}\right)$ e carbonato de sódio $\left(\mathrm{Na}_{2} \mathrm{CO}_{3}\right)$, numa solução hidroalcoólica, utilizado o método descrito por Srivastava et al. (2005) [26] (Esquema 1).

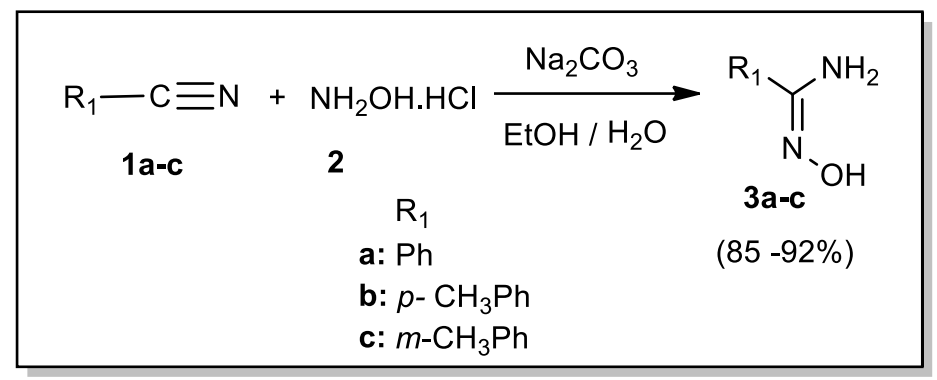

Esquema 1: Sintese das arilamidoximas 3a-c.

Convém destacar que as arilamidoximas obtidas apresentaram bons rendimentos reacionais que variaram de 85 a $92 \%$. Uma vez que as amidoximas são bastante descritas na literatura, sua caracterização foi feita somente através do espectro de infravermelho. As análises dos espectros de infravermelho das arilamidoximas mostraram a ausência da banda característica das nitrilas em $2240 \mathrm{~cm}^{-1}$ do estiramento da ligação $\mathrm{C} \equiv \mathrm{N}$ e o aparecimento de bandas nas regiões de $3498 \mathrm{~cm}^{-1}$, $3366 \mathrm{~cm}^{-1}$ e $1667 \mathrm{~cm}^{-1}$ referente ao estiramento simétrico e assimétrico dos grupos $\mathrm{OH}, \mathrm{NH}_{2}$ e $\mathrm{C}=\mathrm{N}$ respectivamente, o que indica a formação das arilamidoximas 3a-c. A Figura 3 mostra o espectro de IV do composto $\mathbf{3 b}$.

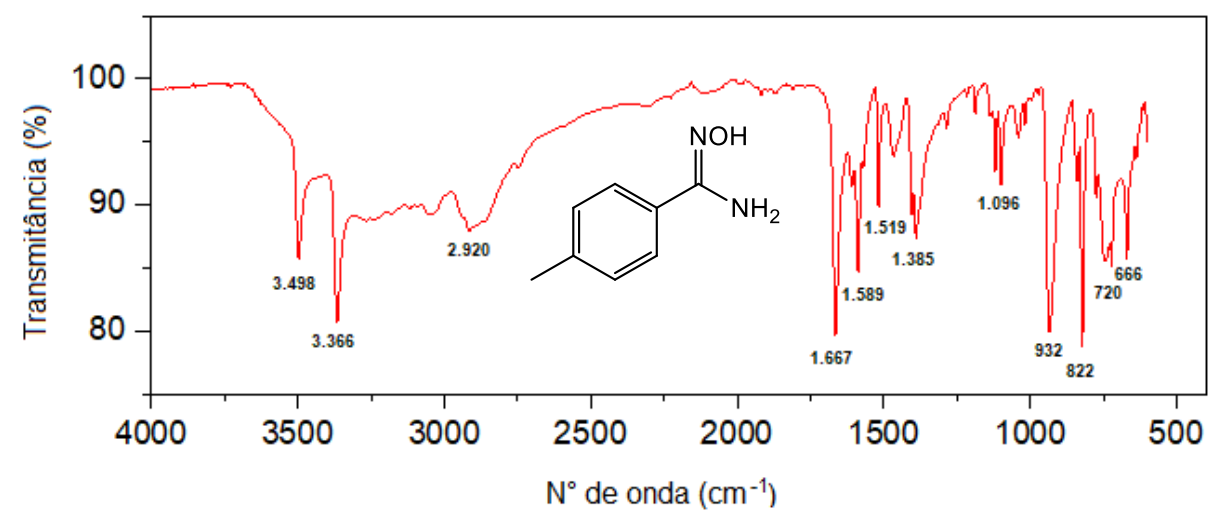

Figura 3: Espectros de IV em pastilha de $\mathrm{KBr}$ do composto $\mathbf{3 b}$.

A síntese dos 1-(3-aril-1,2,4-oxadiazol-5-il) propano-2-ona (5a-c), foram realizadas através da reação entre as arilamidoximas (3a-c) e acetoacetato de etila (4) sob aquecimento e sem utilização de solvente, fornecendo os compostos em rendimentos satisfatórios, ou seja, variando de $60-88 \%$ conforme esquema 2. 


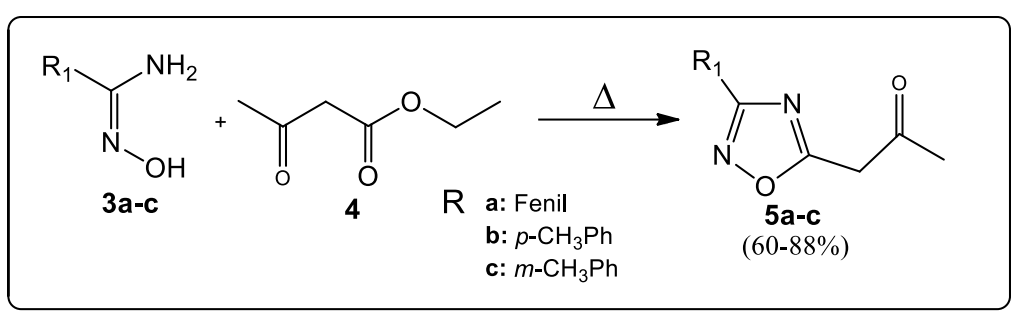

Esquema 2: Síntese de 1,2,4-oxadiazóis 5a-c.

Os 3-aril-(1,2,4-oxadiazol-5-il) propano-2-ona (5a-c), foram obtidos na forma de um óleo amarelado. A caracterização estrutural dos compostos 5a-c foi realizado por meio das técnicas espectroscópicas de infravermelho, $\mathrm{RMN}{ }^{13} \mathrm{C} \mathrm{e}{ }^{1} \mathrm{H}$. No espectro de infravermelho do composto $\mathbf{5 b}$ (Figura 4) é possível observar o estiramento em $1.162 \mathrm{~cm}^{-1}$ referente a ligação C-N.O estiramento da ligação C-O normalmente aparece em $1.271 \mathrm{~cm}^{-1}$, no entanto devido à conjugação do oxigênio com o anel esta banda de absorção que dá a ligação características de dupla ligação ela e movida para uma maior energia aparecendo em $1.362 \mathrm{~cm}^{-1}$. Em $1.591 \mathrm{~cm}^{-1}$ podemos observar a absorção da ligação $\mathrm{C}=\mathrm{N}$ bem como $\mathrm{C}=\mathrm{C}$ que por serem estiramentos fracos aparecem sobreposto justificando assim o alargamento da banda de absorção. $\mathrm{O}$ estiramento agudo e forte de carbonila em $1.718 \mathrm{~cm}^{-1}$ característica de ligação $\mathrm{C}=\mathrm{O}$. A absorção em $2.968 \mathrm{~cm}^{-1}$ característica da ligação $\mathrm{C}-\mathrm{H} s p^{3}$.

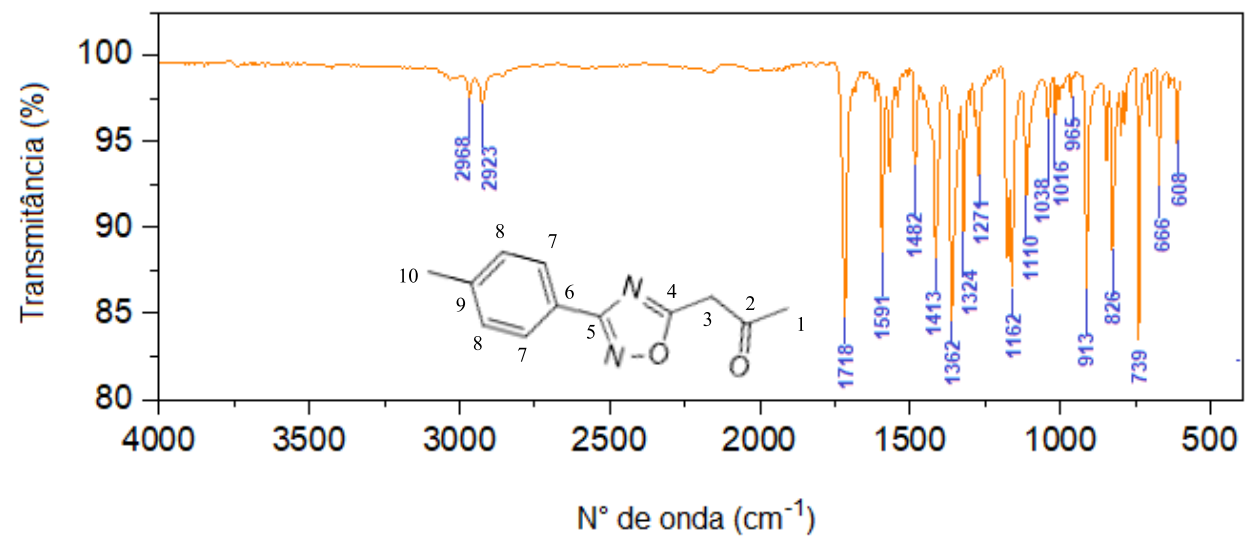

Figura 4: Espectros de IV em pastilha de KBr do composto 5 b.

$\mathrm{Na}$ análise do espectro de $\mathrm{RMN}{ }^{1} \mathrm{H}$ (Figura 5) do composto $\mathbf{5 b}$, em campo alto, são observados três simpletos: o mais protegido em $\delta 2,26 \mathrm{ppm}$ foi atribuído aos hidrogênios da metila $\mathrm{H}-1$; aquele em $\delta$ 2,32 ppm foi atribuído ao $\mathrm{H}-10$ da metila próxima ao anel aromático e o mais deslindado em $\delta 4,10 \mathrm{ppm}$ corresponde aos hidrogênios metilênicos H-3. Os simpletos das metilas apresentaram integrações para três hidrogênios cada e o hidrogênio metilênico para dois. $\mathrm{Na}$ região dos aromáticos observa-se o deslocamento de hidrogênios do sistema AA'BB', apresentando um dubleto em $\delta 7,87 \mathrm{ppm}$ com integração para dois hidrogênios correspondentes ao H-7 cuja constante de acoplamento é $J_{3}=8,1 \mathrm{~Hz}$; estes hidrogênios acoplam com H-8 que aparecem como duplo dubleto em $\delta 7,20 \mathrm{ppm}$ com integração para dois hidrogênios e constantes de acoplamento $J_{3}=8,1 \mathrm{~Hz}$. Observa-se que $\mathrm{H}-8$ possui outra constante de acoplamento com $\mathrm{H}-7$ com valor de $J_{6}=0,9 \mathrm{~Hz}$, indicando que estes hidrogênios são quimicamente equivalentes, mas magneticamente diferentes. 


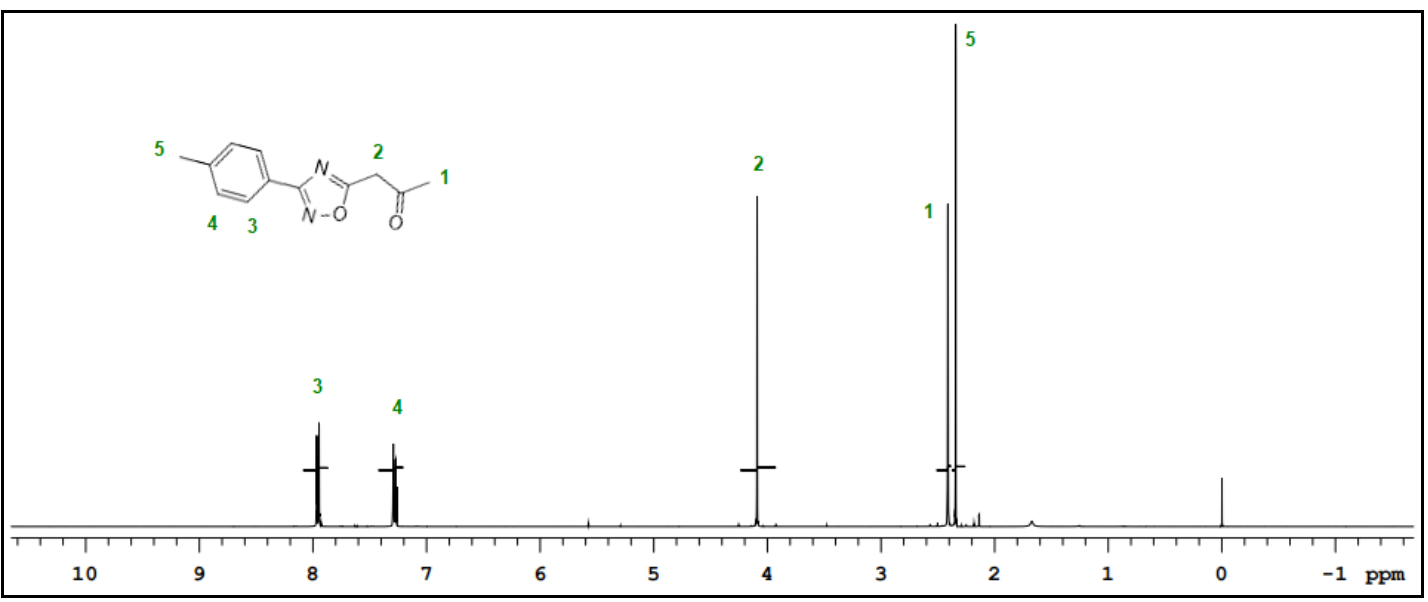

Figura 5: Espectro de $\mathrm{RMN}$ de ${ }^{1} \mathrm{H}(300 \mathrm{MHz})$ em $\mathrm{CDCl}_{3}$ do composto $\mathbf{5 b}$.

Por outro lado, o espectro de $\mathrm{RMN}^{13} \mathrm{C}$ do composto $\mathbf{5 b}$ mostrou os nove núcleos com deslocamento dentro do esperado como podemos observar no espectro da figura 6. Sinais característicos de carbonos aromáticos aparecem entre $\delta 123,61-\delta 141,67 \mathrm{ppm}$. Os carbonos do anel oxadiazólíco C-4 em $\delta$ 168,64 ppm e C-5 $\delta$ 172,91 ppm estão deslindados, e apresentam uma forte interação com grupos eletronegativos. O C-2 possui o $\delta 198,87 \mathrm{ppm}$ por ser um carbono carbonílico. O carbono C-3 aparece na região $\delta 41,51 \mathrm{ppm}$, típica de carbono sp e o C-1 em campo alto com $\delta 21,50 \mathrm{ppm}$.

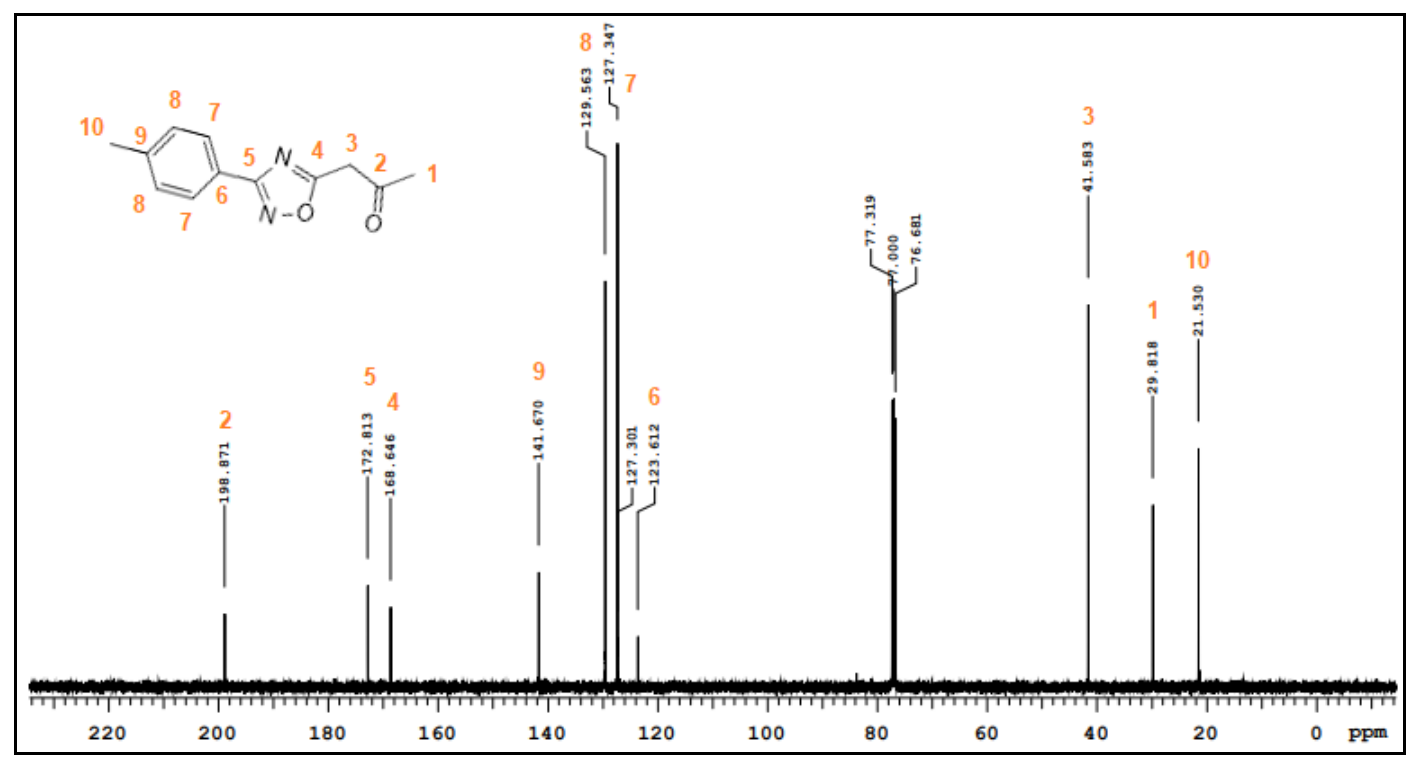

Figura 6: Espectro de $\mathrm{RMN} \mathrm{de}^{13} \mathrm{C}(75 \mathrm{MHz})$ em $\mathrm{CDCl}_{3}$ do composto 5 b.

Os dados espectroscópicos de RMN ${ }^{1} \mathrm{H}$ e $\mathrm{RMN}{ }^{13} \mathrm{C}$ corroboram com os valores encontrados na literatura para o 1-(3-aril-1,2,4-oxadiazol-5-il) butan-2-ona descrito por Freitas et al. (2007) [27]. Em materiais e métodos são descritos os valores dos deslocamento e constantes de acoplamento do espectro de $\mathrm{RMN}{ }^{1} \mathrm{H}$ e $\mathrm{RMN}{ }^{13} \mathrm{C}$.

A reação de redução dos compostos 5a-c com borohidreto de sódio em metanol a $0{ }^{\circ} \mathrm{C}$, resultou na formação dos compostos 6a-c, em rendimentos de 75-88\%, conforme esquema 3. 


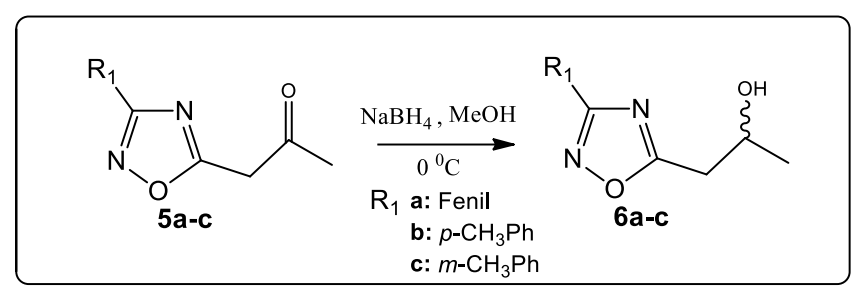

Esquema 3: Reação de redução dos 1,2,4-oxadiazóis 6a-c.

A determinação estrutural dos 3-aril-(1,2,4-oxadiazol-5-il) propan-2-ol foram realizadas através de técnicas espectroscópicas de infravermelho e $\mathrm{RMN}{ }^{1} \mathrm{H}$ e RMN ${ }^{13} \mathrm{C}$. No espectro de IV é possível observar o aparecimento da banda larga de estiramento característica de álcool em 3.406 $\mathrm{cm}^{-1}$ e o desaparecimento da banda característica carbonila em $1.700 \mathrm{~cm}^{-1}$ dos compostos (6a-c). Os valores dos deslocamento químico e constantes de acoplamento do espectro de RMN ${ }^{1} \mathrm{H}$ e RMN ${ }^{13} \mathrm{C}$ estão sumariadas na tabela 1.

Tabela 1: Dados de RMN de hidrogênio e Carbono-13 dos compostos 6a-c.

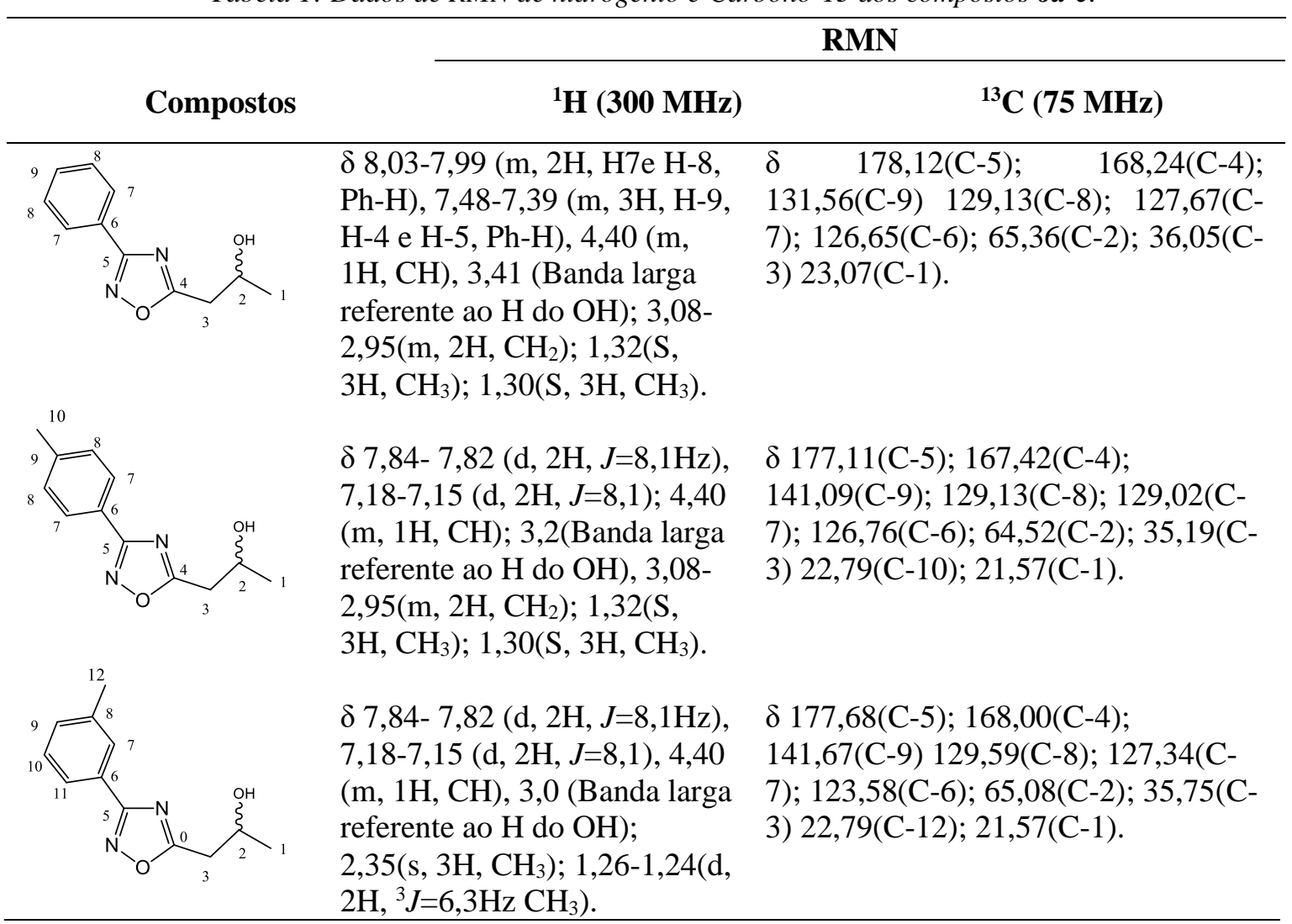

Uma vez sintetizados e caracterizados os compostos 5a-c e 6a-c, estes foram submetidos a uma avaliação da atividade antitumoral frente a três linhagens de células tumorais humanas, sendo elas: HT29 (carcinoma de cólon - humano), NCI H-292 (câncer de pulmão - humano) e HL 60 (Leucemia Promielocitica) pelo Método MTT (Microcultura de Tetrazolium). Dentre os compostos testados o $\mathbf{5 c}$ foi o que apresentou maior percentual de inibição de crescimento celular frente a linhagem HL-60, enquanto os compostos $\mathbf{5 a} / \mathbf{c}$ apresentaram atividade moderada com $50 \%$ e $62,5 \%$ de inibição de crescimento para as HL-60. Por outro lado, o composto $\mathbf{5 b}$ apresentou atividade moderada com 57,1 de inibição de crescimento para as NCI. Os demais compostos tiveram pouco ou nenhuma atividade para as linhagens de células testadas como mostra a tabela 2 . 
Tabela 2: Percentual de inibição do crescimento celular (IC\%) das amostras em dose única de $25 \mu \mathrm{g} / \mathrm{mL}$.

\begin{tabular}{cccc}
\hline & \multicolumn{3}{c}{ Linhagens } \\
\cline { 2 - 4 } Compostos & $\begin{array}{c}\boldsymbol{H T 2 9} \\
\text { \% inibição }\end{array}$ & $\begin{array}{c}\boldsymbol{N C I} \\
\text { \% inibição }\end{array}$ & $\begin{array}{c}\boldsymbol{H L 6 0} \\
\text { \% inibição }\end{array}$ \\
\hline $\mathbf{5 a}$ & 18,6 & 25,4 & 62,5 \\
$\mathbf{5 b}$ & 8,2 & 57,1 & 50,0 \\
$\mathbf{5 c}$ & 32,1 & 39,0 & 84,8 \\
$\mathbf{6 a}$ & 16,7 & 24,0 & 0 \\
$\mathbf{6 b}$ & 18,4 & 14,3 & 0 \\
$\mathbf{6 c}$ & 15,7 & 12,2 & 0 \\
\hline
\end{tabular}

\section{CONCLUSÃO}

Neste trabalho, foi realizada a síntese de três arilamidoximas, intermediários na síntese de 1,2,4-oxadiazóis, em rendimentos de 85-92\%. Os 3-aril-[1,2,4-oxadiazol-5-il]-propan-2-ona (5ac) foram obtidos, em rendimento de $74-88 \%$, recorrendo à metodologia de aquecimento sem uso de base e solvente. Os novos 3-aril-[1,2,4 -oxadiazol-5-il]-propan-2-ol (6a-c) foram obtidos, em bom rendimento $75-88 \%$, através da reação de redução com borohidreto de sódio em metanol dos compostos 5a-c. Todos os compostos foram caracterizados por espectroscopia de IV, RMN de ${ }^{1} \mathrm{H}$ e ${ }^{13} \mathrm{C}$. Por exemplo, os compostos apresentaram banda de absorções de IV em $1592(\mathrm{C}=\mathrm{N})$ e $1361(\mathrm{C}-\mathrm{O})$ e $1720(\mathrm{C}=\mathrm{O}) \mathrm{cm}^{-1}$ (compostos 5a-c) e $3400(\mathrm{OH}) \mathrm{cm}^{-1}$ (compostos 6a-c). Os espectros de $\mathrm{RMN}{ }^{1} \mathrm{H}(300 \mathrm{MHz})$ dos compostos 5a-c mostraram simpleto em $\delta$ 2,25 ppm para o grupo metila e um simpleto em $\delta 4,10 \mathrm{ppm}$ para o grupo. Outros sinais foram observados em locais apropriados. Os espectros RMN ${ }^{13} \mathrm{C}$ apresentaram dois sinais para $\mathrm{NCO}$ e $\mathrm{NCN}$ do anel oxadiazólico na região de $\delta 179,7$ e 167,9 para os compostos 5a-c e 6a-c.

Também foram avaliadas as atividades antitumorais dos compostos $\mathbf{5 a - c , ~ 6 a - c ~ e ~ f r e n t e ~ a ~ t r e ̂ s ~}$ linhagens de células tumorais humanas, sendo elas: HT29 (carcinoma de cólon - humano), NCI H-292 (câncer de pulmão - humano) e HL 60 (Leucemia Promielocitica) pelo Método MTT (Microcultura de Tetrazolium). Dentre os compostos testados o $\mathbf{5 c}$ foi o que apresentou maior percentual de inibição de crescimento celular frente a linhagem HL-60, enquanto os compostos $\mathbf{5 a} / \mathbf{c}$ apresentaram atividade moderada com $50 \%$ e $62,5 \%$ de inibição de crescimento para as HL60.

\section{AGRADECIMENTOS}

Os autores deste trabalho agradecem a todas as agências de fomento que financiaram a nossa pesquisa: CAPES, CNPq e FACEPE pelas bolsas concedidas.

\section{REFERÊNCIAS BIBLIOGRÁFICAS}

1. Bird CW. Heteroaromati city, 5, a unified aromaticity index. Tetrahedron. 1992;48(2):335-340, doi: 10.1016/S0040-4020(01)88145-X.

2. Pace A, Pierro P. The new era of 1,2,4-oxadiazole. Org Biomol Chem. 2009;7(21):4337-48, doi: 10.1039/b908937c.

3. Carbone M, Li Y, Irace C, Mollo E, Castelluccio F, di Pascale A, Cimino G, Santamaria R, Yue-Wei G, Gavagnin M. Structure and cytotoxicity of phidianidines A and B: first finding of 1,2,4-oxadiazole system in a marine natural product. Org Lett. 2011;13(10):2516-19, doi: 10.1021/ol200234r.

4. Freitas JJR, Silva EE, Regueira JLLF, de Andrade AS, Calvalcante PMM, Oliveira RN, Freitas Filho JR. 1,2,4-Oxadiazóis: síntese e aplicações. Rev Virtual Quim. 2012;4(6):670-91, doi: 10.5935/19846835.20120051.

5. Yatam S, Gundla R, Jadav SS, Pedavenkatagari N, Chimakurthy J, Rani N, Kedam T. Focused library design and synthesis of 2-mercapto benzothiazolelinked 1,2,4-oxadiazoles as COX-2/5-LOX inhibitors. J Mol Struct. 2018;(1159):193-04, doi 10.1016/j.molstruc.2018.01.060. 
6. Zhang P, Chen J.H, Dong X, Tang MT, Gao LY, Zhao G S, Yu L.G, Guo XL. 6r, a novel oxadiazole analogue of ethacrynic acid, exhibits antitumor activity both in vitro and in vivo by induction of cell apoptosis and S-phase arrest. Biomed Pharmacother. 2013;(67):58-65, doi: 10.1016/j.biopha.2012.10.011.

7. Bartolozzi A, Abeywardane A, Bosanac T, Broadwater J, Chen Z, Hutzler J, Huber J, Nemoto P, Olague A, Riether D, Simpson T, Takahashi H, Wu L, Zhang Y, Zindell, R. Discovery and optimization of oxadiazole-based FLAP inhibitors. Bioorg Med Chem Lett. 2017;27(20):4652-659, doi: 10.1016/j.bmcl.2017.09.007.

8. Zahanich I, Kondratov, I, Naumchyk V, Kheylik Y, Platonov M, Zozulya S, Krasavin M. Phenoxymethyl 1,3-oxazoles and 1,2,4-oxadiazoles as potent and selective agonists of free fatty acid receptor 1 (GPR40). Bioorg Med Chem Lett. 2015;25(16):3105-111, doi: 10.1016/j.bmcl.2015.06.018.

9. Maftei CV, Fodor E, Jones PG, Daniliuc CG, Franz MH, Kelter G, Fiebig HH, Tamm M, Neda I. Novel 1,2,4-oxadiazoles and trifluoromethylpyridines related to natural products: synthesis, structural analysis and investigation of their antitumor activity. Tetrahedron. 2016;(72):1185-199, doi: 10.1016/j.tet.2016.01.011.

10. Zhan J, Li J-C, Song J-L, Cheng Z-Q, Sun J-Z, Jiang C-S. Synthesis and evaluation of coumarin/1,2,4oxadiazole hybrids as selective BChE inhibitors with neuroprotective activity. J Asian Nat Prod Res. 2019;21(11):1090-103. doi: 10.1080/10286020.2018.1492566.

11. Rasool I, Ahmad M, Khan ZA, Mansha A, Maqbool, T.; Zahoor, A. F.; Aslam, S. Recent advancements in oxadiazole-based anticancer agents. Trop J Pharm Res. 2017;16(3):723-33, doi: 10.4314/tjpr.v16i3.30.

12. Gobe, M, Tomasic T, Markovic T, Mlinaric-Rascan I, Dolenc MS, Jakopin Z. Antioxidant and antiinflammatory properties of 1,2,4-oxadiazole analogs of resveratrol. Chem Biol Interact. 2015;(240): 200-07, doi: 10.1016/j.cbi.2015.08.018.

13. Zhao C, Liu Z.-Q, Diaryl-1,2,4-oxadiazole antioxidants: Synthesis and properties of inhibiting the oxidation of DNA and scavenging radicals Biochimie. 2013;95(4):842-49, doi: 10.1016/j.biochi.2012.12.005.

14. Karad SC, Purohit VB, Thummar RP, Vaghasiya, BK, Kamani RD, Thakor P, Thakkar VR, Thakkar SS, Ray A, Raval DK. Synthesis and biological screening of novel 2-morpholinoquinoline nucleus clubbed with 1,2,4-oxadiazole motifs. Eur J Med Chem. 2017;(126):894-909, doi: 10.1016/j.ejmech.2016.12.016.

15. Jiang C-S, Fu Y, Zhang L, Gong J-X, Wang Z-Z, Xiao W, Zhang H-Y, Guo Y-W. Synthesis and biological evaluation of novel marine-derived indole-based 1,2,4-oxadiazoles derivatives as multifunctional neuroprotective agents. Bioorg Med Chem Lett. 2015;(25):216-20, doi: 10.1016/j.bmcl.2014.11.068.

16. Zhang J, Li J-C, Song J-L, Cheng Z-Q, Sun, J-Z, Jiang, C-S. Synthesis and evaluation of coumarin/1,2,4-oxadiazole hybrids as selective BChE inhibitors with neuroprotective activity. J Asian Nat Prod Res. 2019;21(11):1090-103. doi: 10.1080/10286020.2018.1492566.

17. Parra M, Hidalgo P, Elgueta EY. Synthesis and mesomorphic properties of oxadiazole esters derived from $(R)$-2-octanol, (S)-2-n-octyloxypropanol and (2S,3S)-2-chloro-3-methylpentanol. Liq Cryst. 2008;35(7):823-32, doi: 10.1080/02678290802211114.

18. Xiong H, Yang H, Lei C, Yang P, Hu W, Cheng G. Combinations of furoxan and 1,2,4-oxadiazole for the generation of high performance energetic materials. Dalton Trans. 2019;48(39):14705-711, doi: $10.1039 / \mathrm{c} 9 \mathrm{dt} 02684 \mathrm{c}$.

19. Yan T, Cheng G, Yang H. 1,2,4-Oxadiazole-bridged polynitropyrazole energetic materials with enhanced thermal stability and low sensitivity. Chem Plus Chem. 2019;84(10):1567-577, doi: 10.1002/cplu.201900454.

20. Suzuki J, Okamura D, Gushikawa T, Hirai K, Ando T. Synthesis and insecticidal activity of 1,2,4 oxadiazole and 1,2,4-thiadiazole derivatives. J Pestic Sci. 2011;36(3):392-401, doi: 10.1584/jpestics.G11-28.

21. Neves Filho RAW, Bezerra NMM, Guedes JM, Srivastava RM. An easy synthesis of 3,5-disubstituted 1,2,4-oxadiazoles from carboxylic acids and arylamidoximes mediated by ethyl chloroformate. J Braz Chem Soc. 2009;20(7):1365-369, doi: 10.1590/S0103-50532009000700023.

22.Yang S, Tian X-Y, Ma T-Y, Dai L, Ren C-L, Mei J-C, Liu X-H, Tan C-X. Synthesis and biological activity of benzamides substituted with pyridine-linked 1,2,4-oxadiazole. Molecules. 2020;25(15):350012, doi: 10.3390/molecules25153500.

23 Mossman T. Rapid colorimetric assay for cellular growth and survival:application to proliferation and cytotoxicity assays. J Immunol Methods. 1983;(65):55-63, doi: 10.1016/0022-1759(83)90303-4. 
24. Skehan P, Storeng R, Scudiero D, Monks A, Mcmahon J, Vistica D, Warren JT, Bodesch H, Kenney S, Boyd MR. New colorimetric cytotoxicity assay for anticancer - drug screening. J Natl Cancer Inst. 1990;82(13):1107-112, doi: 10.1093/jnci/82.13.1107.

25. Berridge MV, Ta AS, Mccoy KD, Wang R. The biochemical and cellular basis of cell proliferation assays that use tetrazolium salts. Biochemica.1996;4:14-19.

26. Srivastava RM, Bezerra NMM, de Oliveira SP, da Silva J R. Synthesis of 3-aryl-5-decapentyl-1,2,4oxadiazoles possessing antiinflamatory and antitumor properties. Il Farmaco. 2005;60(12):955-60, doi: 10.1590/S0103-50532009000700023.

27. Freitas JJR, Freitas JCR, da Silva LP, de Freitas Filho JR, Kimura GYV, Srivastava RM. Microwave-induced one-pot synthesis of 4-[3-(aryl)-1,2,4-oxadiazol-5-yl]-butan-2-ones under solvent free conditions. Tetrahedron Lett. 2007;(48):6195-198, doi: 10.1016/j.tetlet.2007.06.116. 